\title{
Aproximación empírica para el diseño de planes de muestreo simple por atributos bajo inspección normal basados en las normas COVENIN 3133-1:2001 y MIL-STD-105E
}

\section{(Empirical approach for single attributes sampling plans design under normal inspection based on standards COVENIN 3133-1:2001 and MIL-STD-105E)}

\author{
Mairett Rodríguez-Balza ${ }^{1}$, Luis Pérez-Ybarra², William Quintana-Rivero²
}

\begin{abstract}
Resumen:
Se propone una regla empírica para la determinación del tamaño de muestra $n$ a tomar en función del tamaño del lote producido $N$, para un muestreo simple por atributos bajo inspección normal basado en la normas COVENIN 3133-1:2001 y MIL-STD-105E; asimismo, se calculó el número de aceptación $c$ para un nivel de calidad aceptable dado, y se construyeron las curvas características de operación correspondientes. Se obtuvieron dos ecuaciones de regresión potenciales para calcular el tamaño de muestra con ajuste $R^{2}$ de $99,91 \%$ y $99,38 \%$, para tamaños de lote $N \leq 666$ y $N>666$, respectivamente, adicionalmente, se diseñó un plan de muestreo para un lote de tamaño 176.000 unidades con un nivel de calidad aceptable de $0,65 \%$ unidades defectuosas. El plan de muestreo propuesto presentó un tamaño de muestra menor $(n=560)$ al de la norma $(n=800)$, con curva característica de operación muy similar al plan de muestreo más próximo recomendado por la norma $(n=500)$. Se recomienda la aplicación de la aproximación empírica para diseñar planes de muestreos más económicos y que a su vez sean cónsonos con las normas COVENIN 3133-1:2001 y MIL-STD-105E.
\end{abstract}

Palabras clave: tamaño del lote; tamaño de muestra; muestreo por atributos; número de aceptación; curva característica de operación.

\begin{abstract}
:
An empirical rule is proposed for determining the sample size $n$ to be taken depending on the lot produced size $N$ for a single attributes sampling under normal inspection based on the standards COVENIN 3133-1: 2001 and MIL-STD-105E; also, the acceptance number $c$ for a given level of acceptable quality was calculated, and the corresponding operating characteristic curves were constructed. Two potential regression equations were obtained for calculation of sample size to fit $\mathrm{R}^{2}$ of $99.91 \%$ and $99.38 \%$ for lot sizes $N \leq 666$ and $N>666$, respectively, in addition, a sampling plan was designed for a lot size of 176,000 units with an acceptable level of quality of $0.65 \%$ defectives units. The proposed sampling plan presented a smaller sample size $(n=560)$ than the standard $(n=800)$, with operating characteristic curve very similar to the closest sampling plan recommended by the standard $(n=500)$. The application of the empirical approach is recommended for the design of cheaper sampling plans which in turn are consonant with the rules COVENIN 3133-1: 2001 and MIL-STD-105E.
\end{abstract}

Keywords: lot size; sample size; attributes sampling; acceptance number units; operating characteristic curves.

\footnotetext{
${ }^{1}$ Universidad Central de Venezuela, Maracay - Venezuela (mairett.rodriguez@ucv.ve)

${ }^{2}$ Universidad de Carabobo, Maracay - Venezuela (\{lmperez4, wfquintana\}@uc.edu.ve)
} 


\section{Introducción}

La norma COVENIN 3133-0-1997, define al plan de muestreo como un conjunto de reglas por medio de las cuales se inspecciona un lote y se decide su aceptación o su no aceptación. Además, señala que el método de inspección por atributos consiste en examinar un ítem o una característica y clasificarla como "conforme" o "no conforme". La decisión con relación al lote, se toma contando el número de ítems no conformes o de no conformidades hallados en una muestra aleatoria (Comisión Venezolana de Normas Industriales, 1995).

La inspección de materias primas, productos semiterminados o productos terminados, forma parte importante del aseguramiento de la calidad (Pérez López, 1999), aunque su objetivo no es estimar la calidad de los lotes, se puede asegurar con cierta probabilidad, que los productos obtenidos en un proceso, son conformes con los criterios acordados entre el consumidor y el productor.

La norma COVENIN establece que para un determinado tamaño de lote y plan de muestreo, se adjudican un tamaño de muestra a extraer, y adicionalmente, se propone un número máximo de unidades no conformes para un nivel de calidad máximo dado. En la práctica, tales valores están tabulados, en la Tabla 1 de la norma COVENIN 3133-1:2001 se establece que para un nivel de inspección normal II (general), y para lotes de tamaño comprendido entre 150.001 y 500.000 unidades, debe extraerse una muestra de tamaño 800, ver Tabla 2- $A$ de la norma COVENIN 3133-1:2001, en dicha tabla además, se establece que para el nivel de calidad aceptable de $0,65 \%$ el número máximo de unidades no conformes es 10 , es decir, si aparecen 11 o más unidades con defectos críticos en el lote, este debe rechazarse, reprocesarse o destruirse, según sea el caso (Comisión Venezolana de Normas Industriales; 2001). Sin embargo, tanto los tamaños de muestra a extraer como los niveles de calidad tolerables se dan en valores discretos, ver Tablas 1 y 2-A de la Norma COVENIN 3133-1:2001, (Comisión Venezolana de Normas Industriales, 2001; Department of Defense, 1989), más aún, los intervalos que definen los tamaños de muestra a extraer tienden a ser muy amplios y aparecen saltos o "huecos" en la asignación tanto de tamaños de muestra como de los niveles de calidad aceptable (Montgomery, 2004), por esta razón, el objetivo de este trabajo es proponer un ajuste empírico o función de regresión que permita asignar tamaños de muestra en función del tamaño del lote dados por las normas COVENIN 3133-1:2001 y MIL-STD-105E, y a partir del tamaño de muestra obtenido, calcular el número de aceptación para un plan de muestreo por atributos simple bajo inspección normal para un nivel de calidad aceptable dado, adicionalmente se diseñará un plan de muestreo bajo las condiciones descritas para un lote de 176.000 unidades con un nivel de calidad aceptable de $0,65 \%$, a fin de ejemplificar el funcionamiento de la metodología propuesta. 


\section{Materiales y métodos}

Los datos a partir de los cuales se realiza la propuesta corresponden a los tamaños de lote $N$, tamaños de muestra $n$ y niveles de calidad aceptable para el muestreo simple por atributos bajo inspección normal presentes en la norma COVENIN 3133-1:2001 y MIL-STD-105E. Dado que los valores de ambas normas son idénticos, en el resto del trabajo se hará referencia únicamente a la norma COVENIN 3133-1:2001.

Las ecuaciones de regresión y los respectivos gráficos se realizaron utilizando el software MS Excel 2010, el número de unidades no conformes tolerables, las curvas características de operación y de potencia se calcularon utilizando el software Derive 6.0, ambos bajo ambiente Windows.

\section{Resultados y discusión}

\subsection{Tamaño de muestra}

Las primeras tres columnas de la Tabla 1 muestran un resumen de los tamaños de muestra $n$ para el nivel de inspección normal II para un muestreo simple por atributos de la norma COVENIN 3133-1:2001, (Comisión Venezolana de Normas Industriales, 2001; Department of Defense, 1989), en la misma, se evidencia que tanto el tamaño de muestra como la amplitud de los intervalos tienden a aumentar con el tamaño del lote $N$, este comportamiento parece indicar que existe una relación o asociación matemática que define el tamaño de muestra en función del tamaño del lote, sin embargo, tal expresión no aparece definida de forma explícita en la norma, una forma de estimarla es hacer un ajuste empírico apoyándose en el análisis de regresión. Dado que la tasa de crecimiento de los intervalos y el tamaño muestra aumenta con el tamaño del lote, esta ecuación posiblemente sea de naturaleza potencial o exponencial.

Tabla 1. Tamaños de lotes y tamaños de muestra a extraer según la norma COVENIN.

\begin{tabular}{|c|c|c|c|c|c|}
\hline NLote $_{\text {inf }}$ & NLote $_{\text {sup }}$ & $\mathbf{n}$ & $\mathbf{n}_{\text {estimado }}$ & Error & $\%$ Error| \\
\hline 2 & 8 & 2 & 1,94496786 & 0,05503214 & 2,75160694 \\
\hline 9 & 15 & 3 & 3,18980717 & $-0,18980717$ & 6,32690581 \\
\hline 16 & 25 & 5 & 4,76825416 & 0,23174584 & 4,63491678 \\
\hline 26 & 50 & 8 & 8,22754001 & $-0,22754001$ & 2,84425015 \\
\hline 51 & 90 & 13 & 13,0668066 & $-0,06680664$ & 0,51389723 \\
\hline 91 & 150 & 20 & 19,5327967 & 0,46720327 & 2,33601636 \\
\hline 151 & 280 & 32 & 31,9222857 & 0,07771429 & 0,24285715 \\
\hline 281 & 500 & 50 & 50,3813735 & $-0,38137349$ & 0,76274699 \\
\hline 501 & 1200 & 80 & 79,4999124 & 0,50008758 & 0,62510948 \\
\hline 1201 & 3200 & 125 & 116,705363 & 8,29463747 & 6,63570998 \\
\hline 3201 & 10000 & 200 & 182,295148 & 17,7048516 & 8,8524258 \\
\hline 10001 & 35000 & 315 & 297,661964 & 17,3380359 & 5,50413838 \\
\hline 35001 & 150000 & 500 & 526,135044 & $-26,1350437$ & 5,22700873 \\
\hline 150001 & 500000 & 800 & 842,853259 & $-42,8532586$ & 5,35665733 \\
\hline
\end{tabular}

La Figura 1 muestra un primer ajuste para establecer una relación de regresión empírica entre las variables $x$ e $y$, la misma se realizó tomando como valor de la variable independiente $x$ al límite 
superior de los intervalos para los tamaños de los lotes, y como variable dependiente $y$ al tamaño de muestra propuesto por la norma. La variable $x$ se seleccionó de esta manera ya que se desea que el tamaño máximo de muestra a tomar en un intervalo dado coincida en lo posible con el valor máximo de dicho intervalo propuesto por la norma. Dicha gráfica muestra que la relación existente es de naturaleza potencial, y que si bien presenta un excelente ajuste de $R^{2}=96,58 \%$, la tasa de crecimiento tiende a ser mayor para tamaños de lote pequeños, de hasta 500 unidades, denominado subconjunto 1 en la Figura 1, que para tamaños de lote mayores, denominado subconjunto 2 en la Figura 1, si bien los desvíos de los datos de la función de regresión parecen ser pequeños, no debe olvidarse que están medidos en escala logarítmica, por lo cual tales diferencias, desvíos o errores de estimación, se inflan notablemente al aplicar antilogaritmos, por esta razón se propone construir dos ecuaciones de regresión potencial, una para el subconjunto 1, y otra para el subconjunto 2 , a fin de minimizar estas desviaciones. Cabe destacar, que el tamaño del lote $N=500$ es considerado en los cálculos de ambas ecuaciones de regresión para que se tome en cuenta la información de todos los valores de la variable $x$ de forma continua.

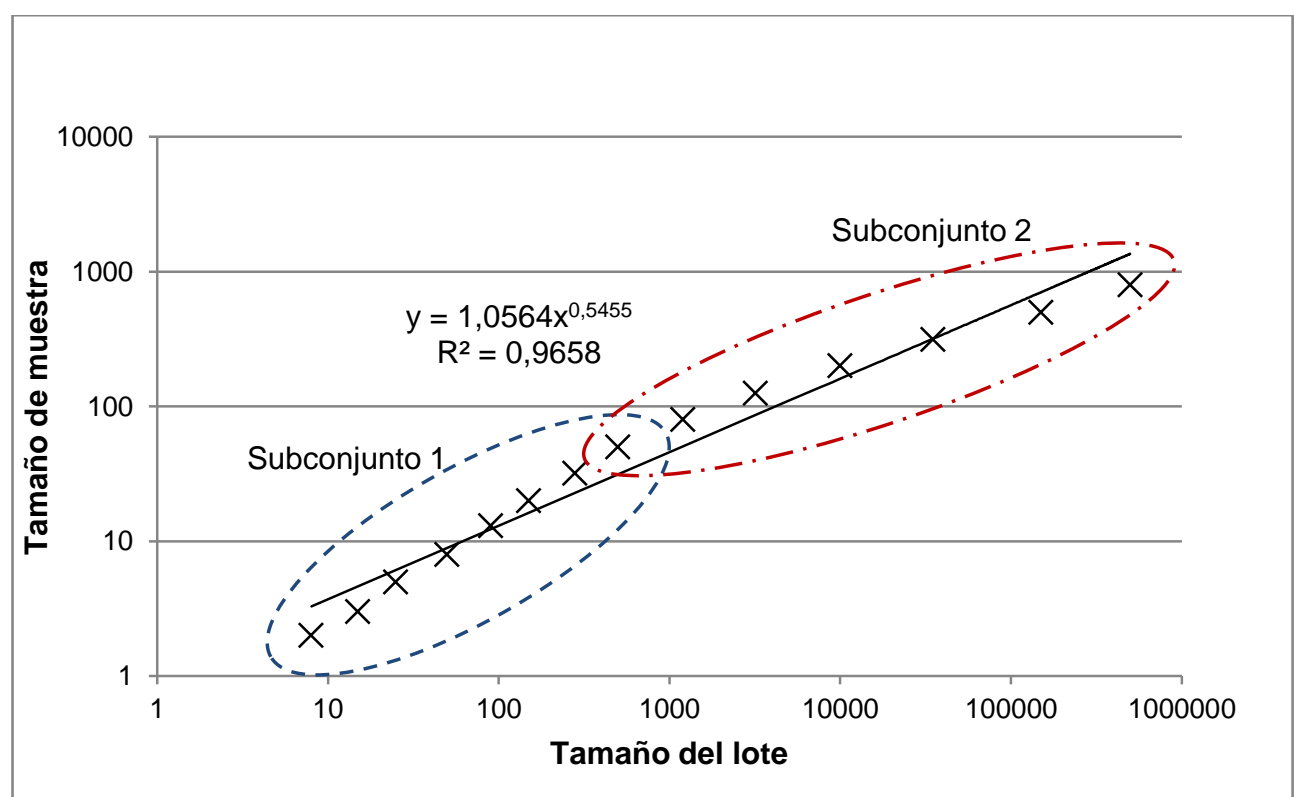

Figura 1. Ecuación de regresión para estimar el tamaño de muestra en función del tamaño del lote. Nota: Ejes en escala logarítmica de base 10.

La Figura 2 muestra las ecuaciones de regresión obtenidas para estimar el tamaño de muestra a extraer en función del tamaño del lote, en la misma se observa que al definir esta función con dos ecuaciones, no solo aumenta el coeficiente de ajuste $R^{2}$ para ambas ecuaciones, sino que además se minimizan las desviaciones de los tamaños de muestra observados con respecto a la línea de tendencia o tamaños de muestra estimados, en las tres últimas columnas de la Tabla 1 se muestran los valores estimados para tales ecuaciones junto con el porcentaje de error relativo de estimación cometido al estimar el tamaño de muestra a extraer en función del tamaño lote, los mismos no difieren en más de $10 \%$ para ninguna de las observaciones tabuladas, lo cual constituye una muy buena aproximación. Es necesario indicar que se definió como error a $e_{i}=n_{i}-\hat{n}_{i}$, y como porcentaje de error relativo a $\% e_{\text {relativo }}=100\left|e_{i}\right| / n_{i}$, siendo $n_{i}$ el tamaño de 
muestra propuesto por la norma COVENIN 3133-1:2001 para el $i$-ésimo límite superior de los intervalos de tamaño de lotes, y $\hat{n}_{i}$ el tamaño de muestra estimado a partir de las ecuaciones de regresión propuestas, ver Tabla 1, columnas 3 a la 6.

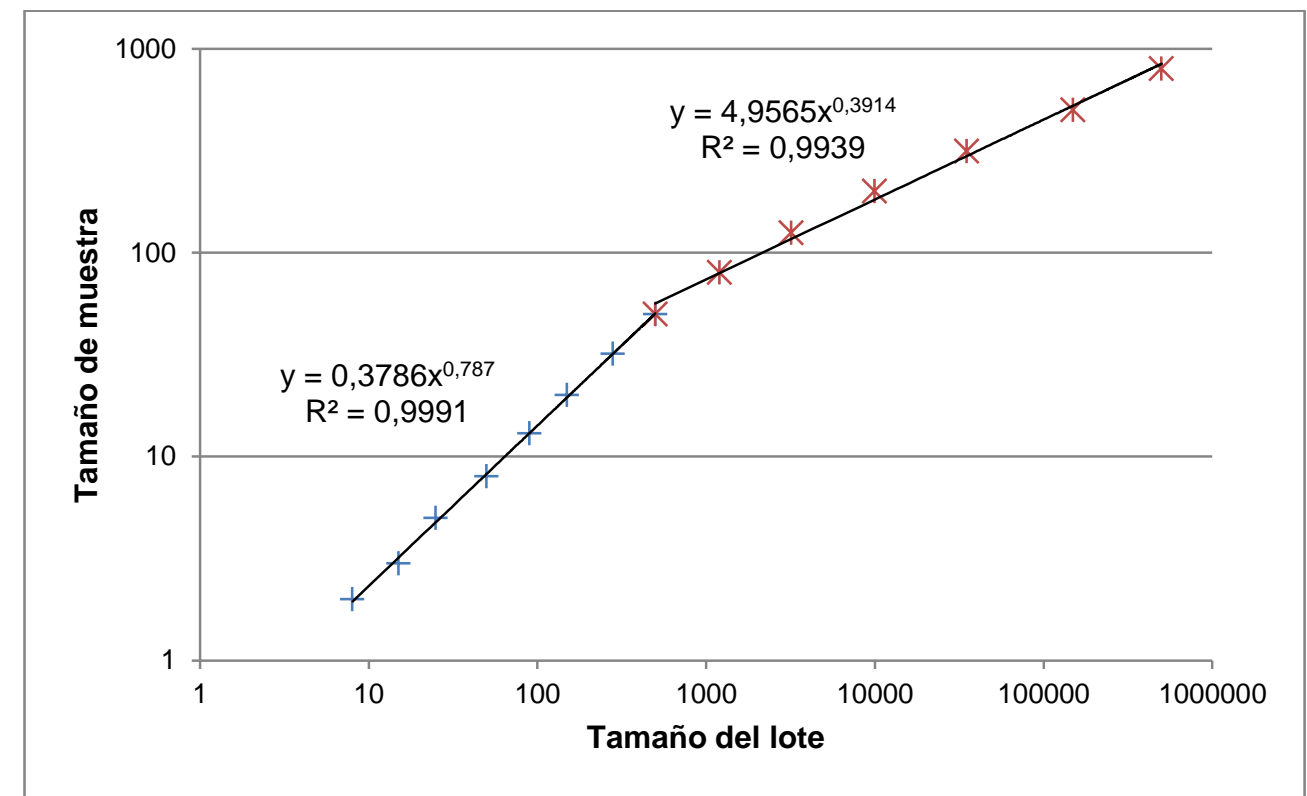

Figura 2. Ecuaciones de regresión para estimar el tamaño de muestra en función del tamaño del lote. Nota: Ejes en escala logarítmica de base 10.

El sistema de ecuaciones formado por las expresiones estimadas mostradas en la Figura 2 se resuelve o se iguala para el tamaño de lote $N=666,3571 \cong 666$, haciendo de esta manera a la función obtenida para estimar el tamaño de muestra $n$ continua en ese punto, esto garantiza que los tamaños de muestra estimados no presenten saltos abruptos y provengan de una función que presenta una gráfica suave, lo cual adicionalmente otorga elegancia a la aproximación empírica desarrollada en este trabajo.

A partir de esta información se propone para este esquema de muestreo la siguiente regla empírica, sea $N$ el tamaño del lote producido y sea $n$ el tamaño de la muestra a ser extraída, este último vendrá dado por:

$$
n=\left\{\begin{array}{cc}
0,3786 N^{0,787} & N \leq 666 \\
4,9565 N^{0,3914} & N>666
\end{array}\right.
$$

Para el caso particular analizado con un tamaño de lote $\mathrm{N}=176.000$ unidades, el tamaño de muestra a extraer por la ecuación (1) quedaría

$$
n=(4,9565)(176.000)^{0,3914}=560,1041 \approx 560 \text { unidades }
$$

El cual es significativamente menor al propuesto por la norma para el ejemplo desarrollado, $n=800$, este resultado no es sorprendente, ya que el tamaño del lote se encuentra más cercano a 150.000 que 500.000 unidades producidas, es de esperarse entonces que el tamaño de muestra 
adjudicado sea un valor más cercano a $n=500$ que a $n=800$, (Comisión Venezolana de Normas Industriales, 2001; Department of Defense, 1989).

\subsection{Número máximo de unidades no conformes toleradas}

Una vez determinado el tamaño de muestra a extraer, se desea calcular cuántos elementos que presenten defectos críticos pueden ser tolerados, es decir, el número de aceptación $c$ suponiendo que la proporción de unidades no conformes del proceso $p_{a d}$ es el adecuado, en ese sentido, sea $n$ el tamaño de muestra, $x$ la cantidad de unidades con defectos críticos, $c \equiv x_{c}$ el número de aceptación o número máximo de objetos con defectos críticos tolerados para el nivel de calidad aceptable $p_{a d}, 1-\alpha$ el nivel de confianza del plan de muestreo y $F(\cdot)$ la función de probabilidad acumulativa, bajo tales condiciones, Montgomery (2004), indica que $x_{c}$ viene dado por

$$
c \equiv x_{c}=\max _{x}\left(\sum_{i=0}^{x}\left(\begin{array}{c}
n \\
i
\end{array}\right) p_{a d}^{i}\left(1-p_{a d}\right)^{n-i} \equiv F\left(x_{c}\right) \leq 1-\alpha\right) ; x, i=0,1, \ldots
$$

Para el caso particular analizado, es decir, para el nivel de calidad aceptable de 0,65\%, $p_{a d}=0,0065$, y para el nivel de confianza de 99\%, la expresión (2) queda

$$
x_{c}=\max _{x}\left(\sum_{i=0}^{x}\left(\begin{array}{c}
560 \\
i
\end{array}\right)(0,0065)^{i}(1-0,0065)^{560-i} \leq 0,99\right)
$$

Utilizando el software Derive 6.0, (Kutzler \& Kokol-Voljc, 2003), se obtiene que para $x=8$, $F(8)=0,9878$, mientras que para $x=9, F(9)=0,9958$, lo cual indica que $x_{c}=8$; es decir, se aceptan hasta 8 unidades con defectos críticos y aún puede considerarse con un nivel de hasta $99 \%$ de confianza que el porcentaje de productos defectuosos del lote es de 0,65\%; por otra parte, de aparecer 9 o más unidades con defectos críticos, se supondrá que el porcentaje de objetos defectuosos en el lote es superior al nivel de calidad aceptable de 0,65\%. Estos resultados son cónsonos con la norma COVENIN 3133-1:2001, ya que $x_{c}$ corresponde un valor que se encuentra a medio camino entre los valores tabulados por esta norma, es decir, este valor crítico es mayor que el establecido por la norma para $n=500$ pero menor al establecido para $n=800$, ver Tabla $2-A$ de la norma COVENIN 3133-1:2001.

Así, el plan de muestreo propuesto para el ejemplo desarrollado en este trabajo queda estructurado de la siguiente manera, para el tamaño del lote considerado de $N=176.000$, extraer una muestra de $n=560$ unidades, y considerar que el nivel de calidad aceptable de 0,65\% unidades con defectos críticos no se cumple si aparecen 9 o más unidades con defectos críticos en la muestra, o dicho de otra forma, se aceptan hasta un total de $x_{c}=8$ unidades con defectos críticos en la muestra. 


\subsection{Curva de potencia y curva característica de operación}

Cytel Software (2010), indica que la función potencia $P(p)$ basada en la distribución binomial, la cual es aplicable al plan de muestreo con proporción de unidades defectuosas o nivel de calidad $p$ del lote para el valor crítico o número de aceptación $x_{c}$, viene dada por

$$
P(p)=\sum_{i=x_{c}+1}^{n}\left(\begin{array}{c}
n \\
i
\end{array}\right) p^{i}(1-p)^{n-i}, 0<p<1
$$

Es decir, corresponde a la probabilidad de rechazar un lote con nivel de calidad o proporción de unidades defectuosas $p$, dado el número de aceptación $x_{c}$. Asimismo, Canavos, (1988), indica que la función característica de operación $C O(p)$ está dada por:

$$
C O(p)=1-P(p)
$$

Para el ejemplo considerado, la función de potencia dada por (3) está dada por:

$$
P(p)=\sum_{i=9}^{560}\left(\begin{array}{c}
560 \\
i
\end{array}\right) p^{i}(1-p)^{560-i}
$$

Los valores para la curva característica de operación dada por (4) se obtienen por diferencia. Note que la expresión (5) contempla una cantidad apreciable de cálculos basados en la distribución binomial para un conjunto de valores $p$ crecientes y cada vez más alejados de la proporción de artículos no conformes del proceso $p_{a d}$.

La Figura 3 muestra las curvas de potencia y curva característica de operación calculadas para el ejemplo desarrollado, $n=560$, y para los tamaños de muestra $n=500$ y $n=800$ mostrados en la norma COVENIN 3133-1:2001; dado que la potencia de una prueba de hipótesis estadística aumenta con el tamaño de muestra si se mantiene fijo el nivel de significación (Cárdenas Castro y Arancibia Martini, 2014), es natural que la potencia de la prueba sea mayor para $n=800$, asimismo, dado que el tamaño de muestra del ejemplo $n=560$ es un valor cercano a $n=500$, no es de extrañar que las curvas de potencia para estos tamaños de muestra sean parecidos, aunque ninguno de los dos es uniformemente más potente, así se observa que inicialmente hay ligeramente más potencia para $n=500$ pero alrededor de $p=0,2$ el comportamiento cambia, esto puede deberse a la naturaleza discreta de la distribución binomial y a la forma en la cual se escoge $x_{c}$, es decir, por aproximaciones sucesivas al nivel de confianza de 99\%, sin embargo, en la práctica, parece natural que las curvas de potencia para los tamaños de muestras de tamaños similares sean similares entre sí, por otra parte, las curvas características de operación, al estar definidas por diferencia, presentan el comportamiento contrario. 

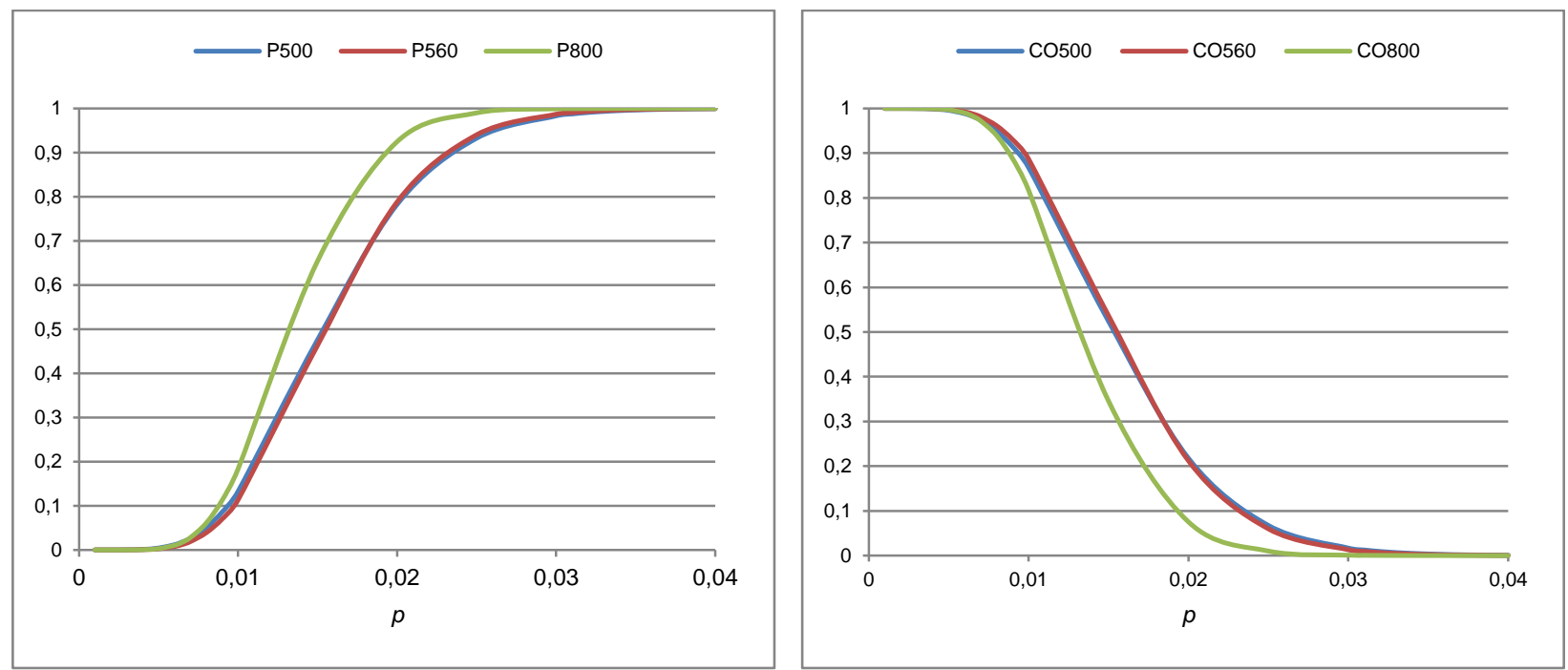

Figura 3. Curvas de potencia y curva característica de operación del ejemplo y para los tamaños de muestra establecidos en la norma. Nota: P500, P560 y P800: Curvas de potencia para $n=500,560$ y 800 , respectivamente. CO500, CO560 y CO800: Curva características de operación para $n=500,560$ y 800 , respectivamente.

\section{Conclusiones y Recomendaciones}

Los autores consideran que esta metodología puede utilizarse para establecer el tamaño de muestreo particularizado para tamaño de lotes y además construir la curva de potencia respectiva, y dado que el tamaño de muestra y los valores críticos son cónsonos con la norma COVENIN 3133-1:2001 y MIL-STD-105E, no se estarían violando estas normas y se podrían diseñar planes de muestreos particularizados, más sencillos y económicos.

\section{Bibliografía}

Canavos, G. C. (1988). Probabilidad y estadística: aplicaciones y métodos. México: McGraw-Hill.

Cárdenas Castro, M. y Arancibia Martini, H. (2014). Potencia estadística y cálculo del tamaño del efecto en G*Power: Complementos a las pruebas de significación estadística y su aplicación en psicología. Salud \& Sociedad, 5(2), 210-224.

Cytel Software. (2010). Statxact 9. User manual. Statistical software for exact nonparametric inference. Cambridge, MA: Cytel Inc.

Kutzler, B. \& Kokol-Voljc, V. (2003). Introduction to Derive 6. A book for learning to use Derive 6 with hints on how to teach with it. Linz, Austria: Kutzler \& Kokol-Voljc OEG.

Montgomery, D. (2004). Control estadístico de la calidad. México: Editorial Limusa S.A. De C.V.

Pérez López, C. (1999). Control estadístico de la calidad: teoría, práctica y aplicaciones informáticas. México: Editorial RA-MA.

USA. Department of Defense. (1989). Military Standard MIL-STD-105E. Sampling procedures for inspection by attributes. Washington, DC: Department of Defense. 
Venezuela. Comisión Venezolana de Normas Industriales. (2001). NORMA COVENIN 31331:2001. Procedimientos de muestreo para inspección de atributos. Parte 1: Esquemas de muestreos indexados por nivel de calidad de aceptación (NCA) para inspección lote por lote. Caracas: FONDONORMA.

Venezuela. Comisión Venezolana de Normas Industriales. (1995). NORMA COVENIN-ISO: 90044:1995. Gestión de la calidad y elementos del sistema de la calidad. Parte 4: Lineamientos para el mejoramiento de la calidad. Caracas: FONDONORMA. 